\title{
Crosstalk Impact on the Performance of Wideband Multicore-Fiber Transmission Systems
}

\author{
Ruben S. Luís (®) Member, IEEE, Georg Rademacher ${ }^{\circledR}$, Senior Member, IEEE, \\ Benjamin J. Puttnam ${ }^{\circledR}$, Member, IEEE, Daniel Semrau ${ }^{\circledR}$, Member, IEEE, Robert I. Killey, Senior Member, IEEE, \\ Polina Bayvel, Fellow, IEEE, Yoshinari Awaji, Member, IEEE, and Hideaki Furukawa, Member, IEEE
}

\begin{abstract}
This work presents an evaluation of the crosstalk impact on the signal-to-noise ratio (SNR) of long-distance $\mathrm{C}$ and $\mathrm{L}$ band wavelength-division multiplexing (WDM) systems using homogeneous multicore fibers with weakly coupled cores. It is experimentally shown that the crosstalk-induced SNR penalty is independent of the transmission distance on sufficiently long uniform links. This results from the approximately linear scaling of the noise contributions from amplified spontaneous emission, fiber nonlinearity, and crosstalk with the transmission distance. The crosstalk-induced SNR penalty on C and L band WDM long distance links is experimentally evaluated, showing significant degradation of signals located towards the long edge of the L-band. Up to $3.8 \mathrm{~dB}$ penalty was observed on a $3866 \mathrm{~km}$ link, in agreement with theoretical predictions. We perform a theoretical analysis of the wavelength allocation of densely packed channels for long distance WDM systems with and without the presence of crosstalk. It is shown that these systems favor the use of relatively short transmission wavelength ranges to minimize the crosstalk impact at long wavelengths. This contrasts with systems without crosstalk, which favor the low loss wavelength region of the transmission fiber.
\end{abstract}

Index Terms-Spatial division multiplexing (SDM), multicore fiber (MCF), crosstalk, wavelength-division multiplexing (WDM).

\section{INTRODUCTION}

$\mathbf{S}$ PATIAL-DIVISION multiplexing (SDM) has recently been considered as a promising response to the exponential increase in capacity demand for optical fiber communications networks [1], [2]. SDM fibers carrying multiple spatial channels have consistently shown the potential to enable per-fiber throughput well beyond that of conventional single-core fibers, with reported transmission records above $1 \mathrm{~Pb} / \mathrm{s}$ since 2012 [3].

Though a large number of SDM fiber types has been proposed in the literature, arguably the one that provides the simplest transition from single-core fiber systems is the homogeneous multicore fiber (MCF) with uncoupled cores. The use of these

Manuscript received October 17, 2019; revised January 21, 2020; accepted January 22, 2020. Date of publication February 21, 2020; date of current version March 13, 2020. (Corresponding author: Ruben Luís.)

Ruben S. Luís, Georg Rademacher, Benjamin J. Puttnam, Yoshinari Awaji, and Hideaki Furukawa are with the National Institute of Information and Communications Technology, Koganei 184-8795, Japan (e-mail: rluis@ nict.go.jp; georg.rademacher@nict.go.jp; ben@nict.go.jp; yossy@nict.go.jp; furukawa@nict.go.jp).

Daniel Semrau, Robert I. Killey, and Polina Bayvel are with the Optical Networks Group, University College London, London WC1E 6BT, U.K. (e-mail: uceedfs@ucl.ac.uk; r.killey@ucl.ac.uk; p.bayvel@ucl.ac.uk).

Color versions of one or more of the figures in this article are available online at http://ieeexplore.ieee.org.

Digital Object Identifier 10.1109/JSTQE.2020.2975662
MCFs for transmission of ultra-high capacity signals has been demonstrated in works such as [4] and over long distances using MCF-based optical amplifiers [5]. MCFs generally have multiple cores within a shared cladding. The cores have similar propagation characteristics, which enables the transmission of time-aligned spatial super channels over long distances [1]. As a consequence, it becomes possible to use shared transmission hardware, digital signal processing (DSP) resources [6], and spatial dimension-based modulation and/or coding [7]. With sufficiently weak coupling between cores, it is possible to use passive optical spatial multiplexing and demultiplexing devices to insert and extract optical signals into the fiber without the need for signal processing to undo inter-core crosstalk (XT). This provides the flexibility required for the implementation of efficient optical network structures, compatible with the requirements of existing single-core fiber networks [8].

The main limitation of MCFs for long-distance transmission is perhaps inter-core XT resulting from residual coupling between cores [9]. This phenomenon and its impact on the performance of optical transmission systems has been extensively described in works such as [9]-[12] and was shown to have a strong influence on the spectral efficiency limits [13]-[17]. However, these works have been limited to narrow band systems or the use of wavelength-division multiplexing (WDM) systems spanning the C-band only. As the technology for the development of deployable MCFs is rapidly progressing, an analysis of the XT impact on wideband WDM transmission systems spanning the $\mathrm{C}$ and L bands is necessary. Nevertheless, such investigation requires taking into account phenomena, such as the wavelengthdependence of XT and the impact of stimulated Raman scattering (SRS) not only on MCF XT but on the contributions of amplified spontaneous emission (ASE) noise and nonlinear interference noise (NLIN). With the recent development of analytical models for the rapid estimation of these quantities over wide bandwidths [18], [19], it becomes possible to obtain insights on the design of WDM systems spanning across the $\mathrm{C}$ and $\mathrm{L}$ bands that would otherwise require prohibitive numerical simulation times or extremely complex and costly experiments.

In this paper, we evaluate the XT-induced SNR penalty in long-distance transmission systems using wideband WDM signals. We begin in Section II by providing and experimentally validating a simple analytical model for the XT-induced SNR penalty. It is shown that this penalty is independent on the transmission distance in uniform links, depending mainly on 
the relation between the powers of the XT, ASE noise and NLIN. In Section III we provide an evaluation of the wavelength dependence of the XT across the $\mathrm{C}$ and $\mathrm{L}$ transmission bands. It is experimentally shown that the crosstalk tilt between the short edge of the $\mathrm{C}$ band and the long edge $\mathrm{L}$ band may reach $10 \mathrm{~dB}$, severely degrading signals located at longer wavelengths. We evaluate this degradation experimentally, showing higher than $3 \mathrm{~dB}$ penalty on a $3866 \mathrm{~km}$ link through a 7-core MCF. Finally, we perform a theoretical analysis of the wavelength dependence of the SNR contributions from ASE, NLIN and XT over wideband long distance transmission. We show that XT-limited systems favor the location of the transmission band at relatively short wavelengths to reduce the impact of XT. This differs from systems without XT-impact, which favor the location of the transmission band near the low-loss wavelengths. Final conclusions are outlined in Section IV.

\section{XT-INDUCED SNR PENALTY}

\section{A. Theory}

The work presented in [12] has shown that, for long distance MCF transmission links with high symbol rate signals or in the presence of moderate inter-core skew, the XT impact may be approximated by that of a gaussian noise with constant power, $P_{\mathrm{xt}}$. In this section, the XT impact on the system performance will be addressed, showing theoretically and experimentally that the XT-induced SNR penalty is approximately independent on the transmission distance for uniform links.

Consider the SNR of a signal with average power, $P_{\mathrm{s}}$, in the presence of XT approximated by:

$$
\mathrm{SNR}=\left(\mathrm{SNR}_{0}^{-1}+\mathrm{XT}\right)^{-1},
$$

where $\mathrm{SNR}_{0}$ is the SNR in the absence of $\mathrm{XT}$, which we will assume, reflects the impact of wavelength dependent ASE noise, NLIN and transceiver noise. The term $\mathrm{XT}=P_{\mathrm{xt}} / P_{\mathrm{s}}$ is the $\mathrm{XT}$, defined as the ratio between the total XT power, $P_{\mathrm{xt}}$, and the signal power. We note that XT is wavelength dependent [20]. Nevertheless, for the sake of this approximation, will be assumed constant within the signal bandwidth. For a uniform link with $N$ spans, the SNR in the absence of XT can be approximated by [13], [21]:

$$
\mathrm{SNR}_{0}=\frac{P_{\mathrm{s}}}{N^{1+\epsilon} \eta P_{\mathrm{s}}^{3}+N P_{\text {ase }}+\kappa P_{\mathrm{s}}},
$$

where the three denominator terms on the right hand side are the powers of the NLIN, ASE noise, and transceiver noise contributions, respectively. $\eta$ is the nonlinear interference noise coefficient as defined in [22], and $\epsilon$ is the coherence factor. $P_{\text {ase }}$ is the ASE noise power generated on each span and $\kappa$ quantifies the noise produced by the transmitter and receiver, referred here as transceiver noise [23]. Assuming a weak coupling regime with $P_{\mathrm{xt}} \ll P_{\mathrm{s}}$, the XT power can be described as:

$$
P_{\mathrm{xt}}=N P_{\mathrm{xt}, \mathrm{s}},
$$

where $P_{\mathrm{xt}, s}$ is the XT power introduced per span in the core under analysis. From (1), we can derive the XT-induced SNR

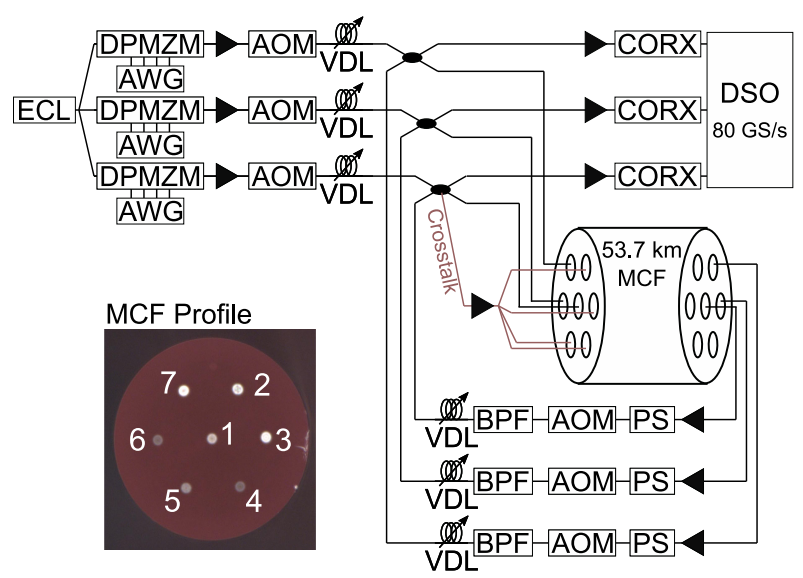

Fig. 1. Experimental setup for estimation of the XT-induced SNR penalty.

penalty similarly to [20] as:

$$
\Delta \mathrm{SNR}=\frac{\mathrm{SNR}_{0}}{\mathrm{SNR}}=1+\mathrm{SNR}_{0} \mathrm{XT},
$$

which explicitly shows that the XT impact also depends on the performance of the system in the absence of XT, becoming stronger for systems operating at high SNR regimes. Extending (4) with (3) and (2) yields the XT penalty as:

$$
\Delta \mathrm{SNR}=1+\frac{P_{\mathrm{xt}, s}}{N^{\epsilon} \eta P_{\mathrm{s}}^{3}+P_{\text {ase }}+\kappa P_{\mathrm{s}} / N} .
$$

For wideband transmission, we have that $\epsilon \approx 0$ [22], resulting in a incoherent accumulation of NLIN. Additionally, if the span count is sufficiently large to neglect the transceiver noise contribution, the penalty can be approximated by:

$$
\Delta \mathrm{SNR} \approx 1+\frac{P_{\mathrm{xt}, s}}{\eta P_{\mathrm{s}}^{3}+P_{\text {ase }}} .
$$

Eq. (6) shows that under the aforementioned conditions, the XT-induced SNR penalty is independent of the transmission distance. Physically, this is an immediate consequence from each noise source scaling with $N$, so the relative strength of all noise sources is independent of the transmission distance. This result has been similarly derived in [20] for the particular case of optimized signal power and worst case XT in a MCF. The following sub-section provides its experimental demonstration.

\section{B. Experiment}

Experimental observation of the XT impact on long distance multicore fiber links has been performed in works such as [24], [25]. However, those works were not devoted specifically to the analysis of the XT penalty but rather to its mitigation using DSP techniques. As such, we will revisit those experiments to estimate the XT-induced performance impact over a long distance link to validate the formulation for the XT-induced penalty shown in the previous sub-section.

Consider the simplified experimental setup shown in Fig. 1. It consisted of 3 recirculating loops running through 3-cores of a $53.7 \mathrm{~km}$ 7-core homogeneous MCF. A $100 \mathrm{kHz}$ linewidth external cavity laser (ECL) operating at $1550 \mathrm{~nm}$ was used as a common light source to produce 3 spatial channels. Each channel was 
modulated by a dual-polarization dual-parallel Mach-Zehnder modulator (DPMZM) driven by 4 arbitrary waveform generators (AWGs) operating a $60 \mathrm{GS} / \mathrm{s}$. The modulations applied to the signals consisted of polarization-multiplexed quadrature phaseshift keying (PDM-QPSK) signals at 20 GBaud produced using $2^{15}-1$ bit pseudo-random binary sequences and root-raised cosine pulse shaping with a roll-off of 0.01. Erbium-doped fiber amplifiers (EDFAs) were used to adjust the signals powers. Variable delay lines (VDLs) were used to match the propagation delays of the signals entering the loops as well as within each loop. Synchronized acousto-optic modulators (AOMs) were used to control the light input into the loops and within each loop. We note that this alignment was not required for the sake of this analysis. Nevertheless, it simplified the experimental setup by using only one timing source for all the AOMs. Also within the loops, we used polarization scramblers (PSs) and band-pass filters (BPFs) to mitigate the impact of polarization-dependent loss and excess ASE noise, respectively. In addition to the 3 main signals, a sample of one of the signals was tapped off to an EDFA and split into the remaining 4 cores of the fiber to induce additional XT. The inset of Fig. 1 shows a profile of the MCF. Six trench-assisted cores were arranged in a ring around a center core within a $160 \mu \mathrm{m}$ diameter cladding. The core pitch and radius were $44 \mu \mathrm{m}$ and $8 \mu \mathrm{m}$, respectively. These properties led to an average inter-core XT of $-42 \mathrm{~dB}$ between pairs of adjacent cores, at a wavelength of $1550 \mathrm{~nm}$. The signals at the recirculating loop output were detected by 3 coherent receivers (CORX) sharing an ECL as a common LO, and detected using a 12-channel digital sampling oscilloscope (DSO) operating at $80 \mathrm{GS} / \mathrm{s}$. DSP was performed offline using MATLAB and C. It consisted of resampling to 2 samples per symbol and normalization stages, followed by frequency-domain dispersion compensation and time-domain $2 \times 2$ multiple input-multiple output modules with 33 -tap equalizers. The equalizers taps were updated using a data-aided least-mean squares algorithm until convergence. Afterwards, we used a decision-directed algorithm. Carrier recovery was performed within the equalizer loop. The SNR was estimated by computing the power of the noise affecting the final signal after the DSP. For this work, we used only the performance of the channel propagating through the center core of the MCF and we compared the performance with and without $\mathrm{XT}$ by enabling or disabling the signals propagating through all the remaining cores.

Fig. 2 shows the dependence of the SNR estimated for a signal propagating through the center core on the launch power with and without the presence of XT for transmission distances of $2685 \mathrm{~km}, 5370 \mathrm{~km}$, and $8055 \mathrm{~km}$. For each transmission reach, it is shown that the optimum launch power remains the same with and without XT, within the $1 \mathrm{~dB}$ power resolution of our measurements. This is in agreement with the results predicted theoretically in [17], [20], [21]. To address the XT-induced penalty, Fig. 3 shows the dependence of the SNR on the transmission distance with and without the presence of XT for the optimum launch power of $-4 \mathrm{dBm}$. The latter was estimated from the results shown in Fig. 2. It is shown the impact of XT on the SNR leads to a nearly constant penalty with the transmission distance, as shown in Fig 3. The XT penalty was

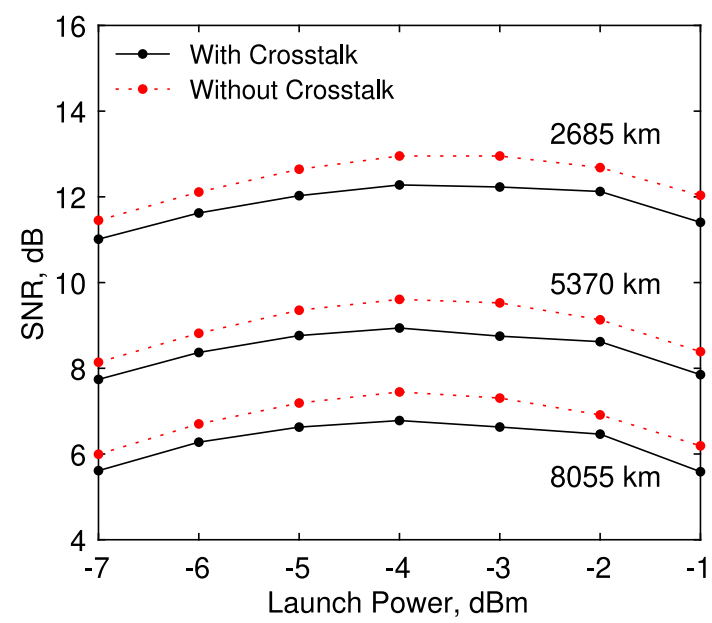

Fig. 2. Dependence of the SNR with and without XT on the launch power for a 7-core MCF link with $2685 \mathrm{~km}, 5370 \mathrm{~km}$, and $8055 \mathrm{~km}$.

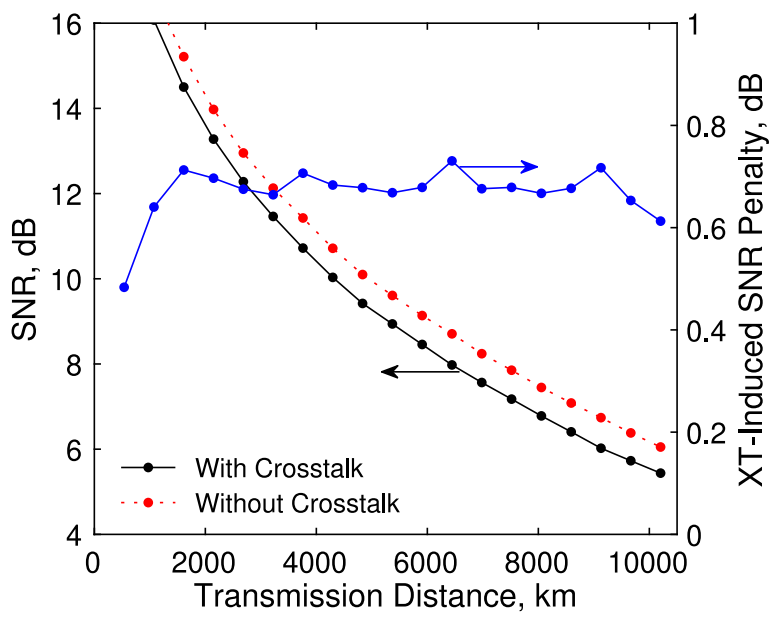

Fig. 3. Dependence of the SNR with and without XT on the transmission distance for a 7-core MCF link, as well as the corresponding XT-induced SNR penalty.

$0.67 \pm 0.06 \mathrm{~dB}$ for transmission reach above $2000 \mathrm{~km}$. We note that this observation is in agreement with the behavior predicted in the previous sub-section with equations (4) and (6) despite the fact that we used a single-channel, limiting the validity of the assumption on the incoherent NLIN accumulation.

We note that the observations reported in this section neglect the fact that the nonlinear interaction between the signal and the MCF material leads to power-dependent XT, as reported in [26]. In this work, we have assumed this to be a valid approach given the relatively low powers used and the fact that in sufficiently long fibers, the XT contributions produced at the MCF sections where the signal power is sufficiently high to enter a nonlinear coupling regime are small in comparison with the XT contributions generated in a linear coupling regime.

\section{XT-Induced Penalty IN WIDEBAnd Systems}

\section{A. XT Wavelength Dependence}

Section II presented a simple model for computing the XT-induced SNR penalty. However, to consider its application 


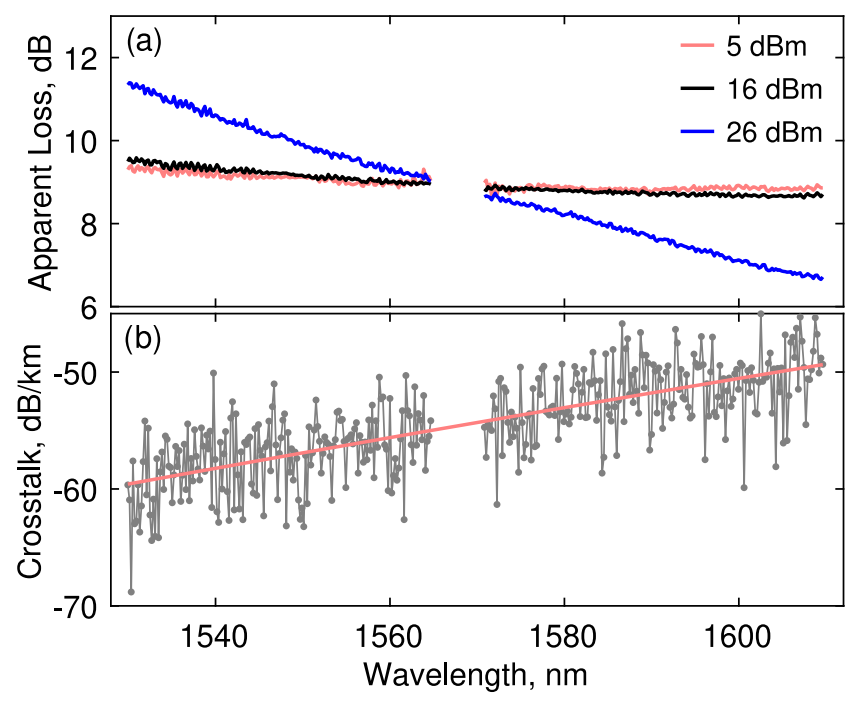

Fig. 4. (a) Apparent loss of a $31 \mathrm{~km} 22$-core MCF for a wideband comb signal with total launch powers of $5 \mathrm{dBm}, 16 \mathrm{dBm}$, and $26 \mathrm{dBm}$. (b) Wavelength dependence of the average XT in the same fiber. The continuous red line is a linear regression acting as a visual guide.

to wideband systems spanning the $\mathrm{C}$ and $\mathrm{L}$ transmission bands, one must take into account the wavelength dependencies of the phenomena degrading the SNR. MCF XT has been shown to be strongly dependent on the operation wavelength, possibly exceeding a $10 \mathrm{~dB}$ range between the short and long edges of the $\mathrm{C}$ and $\mathrm{L}$ bands, respectively [27]. This variation results mainly from the wavelength dependence of the field confinement within each core. The XT wavelength dependence was experimentally addressed in [28] and further extended in [29] to evaluate the impact of stimulated Raman scattering (SRS) on XT measurements and will be revisited here to consider the XT wavelength dependence on wideband transmission system performance.

We considered the transmission of a wideband signal produced using a frequency comb generator, whose output consisted of more than 500 carriers at a frequency spacing of $25 \mathrm{GHz}$ spaning $\mathrm{C}$ and $\mathrm{L}$ bands. The comb signal was depolarized, filtered by optical processors, and amplified using $\mathrm{C}$ and $\mathrm{L}$ band amplifiers to generate a high power signal with $80 \mathrm{~nm}$ bandwidth (360 carriers). The wavelength range spanned from $1530 \mathrm{~nm}$ to $1610 \mathrm{~nm}$, with a $6.5 \mathrm{~nm}$ guard band between $\mathrm{C}$ and $\mathrm{L}$ bands. The wideband signal was launched into one core of a $31 \mathrm{~km} 22$-core MCF with its output and the output of an adjacent core measured using optical spectrum analyzers. Fig. 4(a) shows the apparent loss of the fiber for total launch powers of $5 \mathrm{dBm}, 16 \mathrm{dBm}$, and $26 \mathrm{dBm}$ (or $-20.6 \mathrm{dBm},-9.6 \mathrm{dBm}$, and $0.4 \mathrm{dBm}$ per carrier). The impact of SRS is clearly visible as the launch power is increased. The power transfer from signals located at the short C-band wavelengths to the long L-band leads to a loss tilt of approximately $5 \mathrm{~dB}$ from the short edge of the $\mathrm{C}$ band to the long edge of the L band. Fig. 4(b) shows the average XT measured for each carrier. These measurements were taken over a relatively short period of time of 2 hours, compared to the required to fully characterize the XT statistics as we used unmodulated carriers. Hence the strong variations observed in Fig. 4(b). Nevertheless,
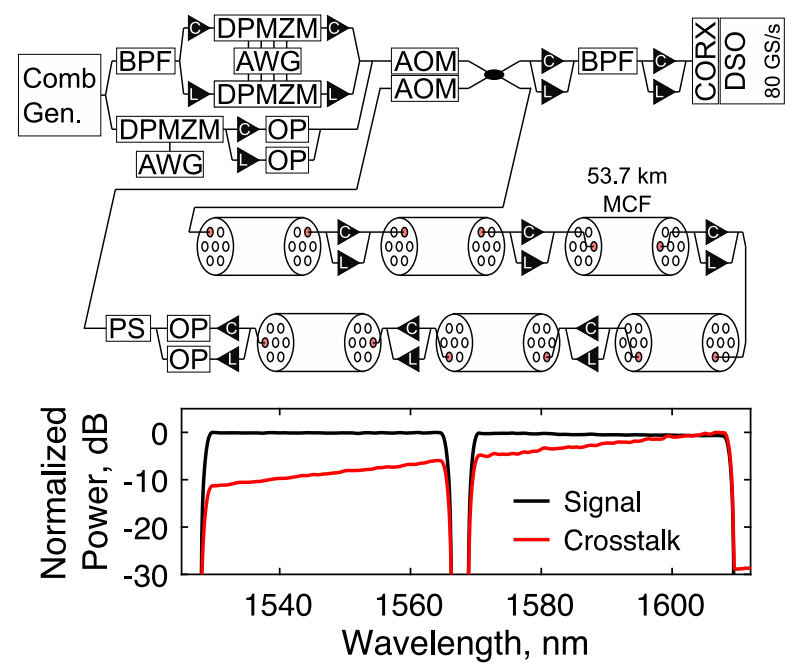

Fig. 5. Simplified experimental setup for SNR measurements on a long distance wideband WDM transmission over 6 cores of a 7-core MCF. The inset shows normalized spectrums of the launched signal and the XT measured on the center core of the MCF. Note that the multiple depictions of the MCF actual represent the same fiber with the signal traversing multiple cores.

the wavelength dependence is clear, with a tilt nearly reaching $10 \mathrm{~dB}$. This is in agreement with the observations in [9].

From these observations, one may assume that the XT cannot be assumed constant with wavelength, particularly for the design of wideband transmission systems spanning $\mathrm{C}$ and $\mathrm{L}$ bands. In principle, systems operating in the L-band will be more significantly degraded by XT. This has been demonstrated in [30], where a recirculating loop experiment was used to show a reduction of 58\% in transmission reach for a signal positioned at the long edge of the L-band with respect to the same signal without significant XT impact. Under the same conditions, a signal positioned at the short edge of the C-band suffered a XT-induced reach reduction of $12.5 \%$. Here, we revisit this experiment to evaluate the wavelength dependent XT-induced penalty.

Consider the simplified experimental setup is shown in Fig. 5. A wideband frequency comb generator capable of producing $25 \mathrm{GHz}$ spaced carriers and an output wavelength range spanning the $\mathrm{C}$ and $\mathrm{L}$ bands was used as light source. Even and odd channels of a sliding 5-channel test band were selected from the comb output using a BPF and modulated by two DPMZMs driven by 4 AWGs. C or L band EDFAs were used to amplify the sliding test band. The remaining carriers at the comb output were modulated by a single DPMZM, driven by one AWG and filtered using optical processors (OP) to form a dummy channel band. The chosen modulation was 24.5 GBaud PDM-QPSK with a root-raised cosine shape and roll-off of 0.01 . The combined test and dummy bands had 389 carriers from $1529.9 \mathrm{~nm}$ to $1607.7 \mathrm{~nm}$ with an approximately $5 \mathrm{~nm}$ guard band between $\mathrm{C}$ and $\mathrm{L}$ bands. This signal was transmitted with an optimum launch power of $22 \mathrm{dBm}$, through a recirculating loop, which consisted of the 6 outer cores of the 7-core MCF described in Section II. The inset of Fig. 5 shows the normalized spectrums of the signal at the transmitter output and the measured XT at the center core of the MCF after 1 span. This crosstalk was 


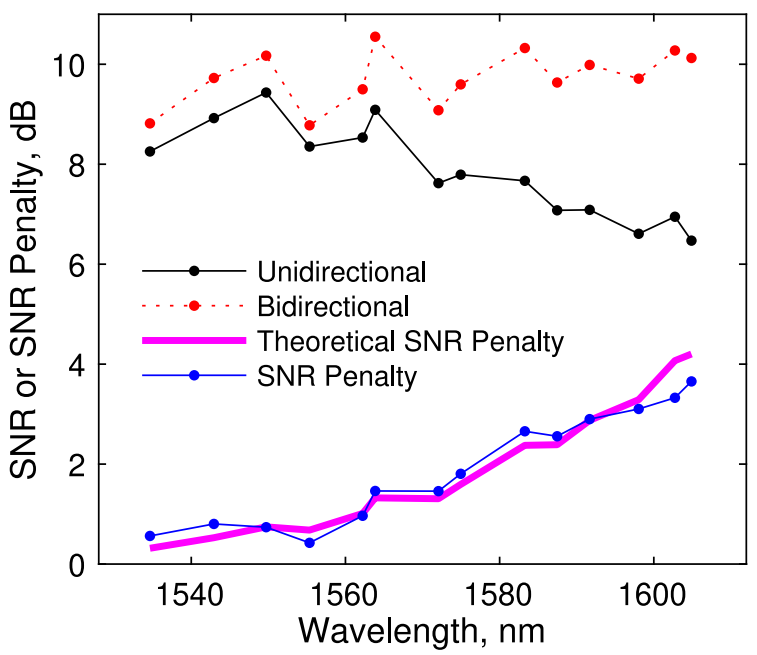

Fig. 6. Wavelength dependence of the SNR after unidirectional (with XT) and bidirectional (reduced XT) transmission through $3866 \mathrm{~km}$ on a 7-core MCF and corresponding XT-induced SNR penalty.

produced by inputting 6 replicas of the WDM signal into the 6 outer cores of the MCF. The receiver was similar to the one described in Section II but using two amplification stages with a $\mathrm{BPF}$ in-between to select the channel under analysis. To evaluate the performance with and without XT, two transmission regimes were considered. When including XT, the signal was input into the outer MCF cores in the same propagation direction. To estimate the performance in the absence of XT, the signals in adjacent cores were input in opposing directions. This technique has been referred to as direction interleaving and is known to substantially reduce the inter-core XT [31].

Fig. 6 shows the wavelength dependence of the measured SNR after $3866 \mathrm{~km}$ with unidirectional and bidirectional transmission and the corresponding SNR penalty, which in this case approximates the XT-induced SNR penalty. It is shown that the SNR penalty increases significantly for wavelengths above $1560 \mathrm{~nm}$. It may be assumed that at this point the XT-impact dominates the system performance, degrading the SNR up to $3.5 \mathrm{~dB}$ at the longest considered wavelength of $1604 \mathrm{~nm}$.

In addition, Fig. 6 shows a theoretical estimate of the SNR penalty due to crosstalk computed using (4). The values of SNR in the absence of crosstalk were approximated using the measured SNR with bidirectional transmission and the crosstalk wavelength dependence was assumed linear with a slope of $0.17 \mathrm{~dB} / \mathrm{nm}$, computed from the measurement in the inset of Fig. 5. This estimate is shown to agree fairly well with our measurements, validating the theoretical approach for long distance transmission systems.

\section{B. XT-Conditioned Wavelength Dependent Performance}

In the previous sub-section, it was shown that the wavelength dependence of XT may condition the performance significantly of long-distance links, particularly for systems using the L-band. In this sub-section, we extend this analysis using analytical and semi-analytical models to evaluate the relative impact of XT, NLIN, and ASE noise on the performance of wideband WDM
TABLE I

SiMULATION PARAMETERS

\begin{tabular}{|c|c|}
\hline Fiber Loss & $\begin{array}{l}0.210 \mathrm{~dB} / \mathrm{km}(1550 \mathrm{~nm}) \\
0.206 \mathrm{~dB} / \mathrm{km}(1575 \mathrm{~nm}) \\
0.230 \mathrm{~dB} / \mathrm{km}(1625 \mathrm{~nm})\end{array}$ \\
\hline Group velocity dispersion $(1550 \mathrm{~nm})$ & $17 \mathrm{ps} / \mathrm{nm} / \mathrm{km}$ \\
\hline Dispersion slope & $67 \mathrm{fs} / \mathrm{nm}^{2} / \mathrm{km}$ \\
\hline Nonlinear index coefficient & $2.6 \times 10^{-20} \mathrm{~m}^{2} / \mathrm{W}$ \\
\hline Raman gain slope & $0.028 \mathrm{~W}^{-1} \mathrm{~km}^{-1} \mathrm{THz}^{-1}$ \\
\hline Raman gain & $0.04 \mathrm{~W}^{-1} \mathrm{~km}^{-1}$ \\
\hline Core radius & $5.5 \mu \mathrm{m}$ \\
\hline Trench radius & $6.5 \mu \mathrm{m}$ \\
\hline Cladding radius & $62.5 \mu \mathrm{m}$ \\
\hline Core pitch & $40 \mu \mathrm{m}$ \\
\hline Core count & 4 \\
\hline Cladding refractive index & 1.4544 \\
\hline$\Delta_{\text {core }}$ & $0.35 \%$ \\
\hline$\Delta_{\text {trench }}$ & $-0.16 \%$ \\
\hline Spool radius & $135 \mathrm{~mm}$ \\
\hline Crosstalk between & $-60 \mathrm{~dB} / \mathrm{km}(1550 \mathrm{~nm})$ \\
\hline adjacent cores & $-52 \mathrm{~dB} / \mathrm{km}(1625 \mathrm{~nm})$ \\
\hline \multicolumn{2}{|l|}{ Transmission Parameters } \\
\hline Signal symbol rate & 25 GBaud \\
\hline Channel spacing & $25 \mathrm{GHz}$ \\
\hline Channel frequency grid & $\begin{array}{l}186.25 \mathrm{THz} \\
\text { to } 196.25 \mathrm{THz}\end{array}$ \\
\hline EDFA noise figure & $5 \mathrm{~dB}$ \\
\hline
\end{tabular}

systems transported through MCFs. For XT modeling, we used the simplified model proposed in [18]. For the computation of the ASE impact, we used a semi-analytical model based on [32], which allows estimating the apparent fiber loss under the impact of SRS. The linear fiber loss was based on interpolating a real measurement of a low-loss single-core fiber, scaled to the typical losses observed in existing MCFs. This interpolation was also shifted to closely match the wavelength dependence of the loss observed in Fig. 4 for the lowest launch power of $5 \mathrm{dBm}$. Finally, the NLIN was computed using the model proposed in [19]. This model takes into account the impact of SRS, accurately predicting the wavelength dependence of NLIN in wideband transmission systems. In addition, we assumed Gaussian modulated signals for all considered cases. Table I details the fiber parameters used for simulation in this work. In this section, the performance analysis will be based on an existing fiber. We have selected the MCF initially presented in [33]. This fiber has 4 cores in a rectangular arrangement within a cladding with a diameter of $125 \mu \mathrm{m}$. Its propagation characteristics, tensile strengh and productivity are similar to those of standard fibers [33]. As such, it is a good candidate for future MCF deployments in long distance links. We assumed the fiber parameters provided in [33], with which the XT model provided an accurate prediction of the average XT at the reference wavelengths of $1550 \mathrm{~nm}$ and $1625 \mathrm{~nm}$.

Fig. 7 shows an example of the wavelength dependence of the SNR and the SNR contributions from ASE, NLIN and XT assuming a WDM signal spanning the $\mathrm{C}$ and $\mathrm{L}$ bands. For this example, we assumed transmission using the 4-core fiber on a $1600 \mathrm{~km}$ link with $80 \mathrm{~km}$ spans and lumped amplification with amplifier noise figures of $5 \mathrm{~dB}$. It is assumed that the gain of the amplifiers perfectly compensates the apparent loss of the fiber, 


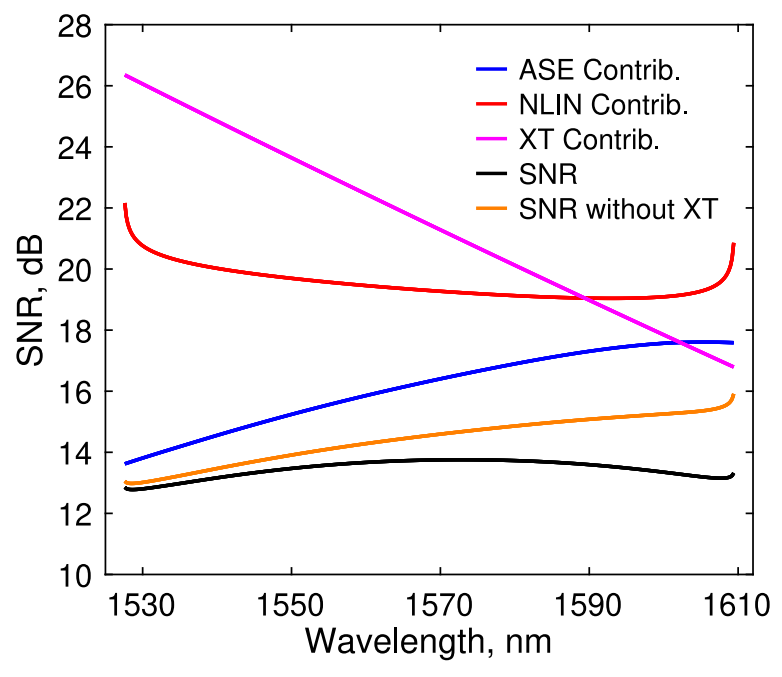

Fig. 7. Theoretical wavelength dependence of the SNR and the SNR contributions from ASE, NLIN, and XT on $1600 \mathrm{~km}$ link with $80 \mathrm{~km}$ spans using the 4-core MCF described in Table I.

leading to a flat spectrum at the input of each span. Alternative amplification schemes could be used to maximize SNR, including power pre-emphasis. However, the use of such schemes in this work would obscure the insights that we intend to convey and may be considered outside its scope. The launch power was computed by maximizing the total throughput assuming Gaussian modulated signals. Fig. 7 also includes the SNR that would be obtained without XT, also with the launch power set to maximize the total throughput. We note that the difference between the optimum launch powers with and without XT was approximately $0.1 \mathrm{~dB}$, in agreement with [21].

Comparing the SNR with and without XT, it is clear that this system is dominated by the impact of ASE noise in the C-band region. When moving towards the L-band, the performance becomes progressively dominated by XT until reaching a penalty higher than $2 \mathrm{~dB}$ at the long edge of the L-band. Note that this behavior is similar to that observed experimentally in the previous sub-section. In the absence of crosstalk, the system performance is dominated by ASE, which has lower impact at longer wavelengths due to SRS. This is in agreement with the observations reported in [34] and suggests that this system favors the use of the L-band for transmission. In contrast, the introduction of XT shifts the highest performing wavelengths to the region corresponding to the short $\mathrm{L}$ band wavelengths, around $1575 \mathrm{~nm}$.

Fig. 7 suggests that in XT-dominated systems, the optimal operating wavelengths may be significantly different to those in non-XT-dominated systems. To evaluate this phenomena, we computed the maximum achievable throughput when transmitting 50,100 or 200 channels densely packed in a $25 \mathrm{GHz}$ grid. Each channel carried Gaussian modulation with $25 \mathrm{GHz}$ bandwidth. We assumed densely packed signals to minimize the required transmission bandwidth of the system, which would lead to a less complex amplifier design. We note that although such a system may not be employed in practice due to other constraints, these assumptions provide an upper bound to the

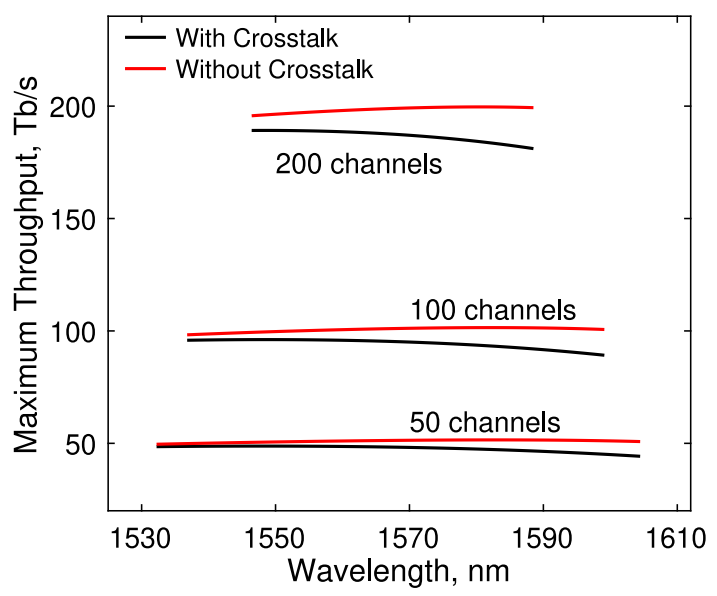

Fig. 8. Theoretical example of the maximum throughput dependence on the center wavelength of the transmission band assuming transmission bands with 50,100 , and 200 channels. This example considered a $1600 \mathrm{~km}$ link using a 4-core MCF with $80 \mathrm{~km}$ spans.

actual throughput achievable in real systems. Fig. 8 shows the maximum throughput when varying the center of the transmission band within the limits of the $\mathrm{C}$ and $\mathrm{L}$ bands. The contributions of ASE noise and fiber nonlinearity to the SNR have been computed independently for each combination of channel count and center wavelength, taking into account the prominence of SRS for each channel count. Despite the relatively small variations of the throughput, it is clear that in the absence of XT the highest performance is achieved for wavelengths around $1580 \mathrm{~nm}$, corresponding to the lowest loss region. In the presence of XT, the optimum wavelengths are substantially lower, positioned the region around $1545 \mathrm{~nm}$.

From Fig. 7, it is clear that a comparison between wideband transmission systems with and without XT requires optimizing the positioning of the transmission band. In that sense, Fig. 9(a) shows the optimal wavelength positions of the transmission bands with and without XT (shaded areas) as a function of the number of channels in the aforementioned densely packed condition. Again, the main distinction between the cases with and without XT is that the latter favor the use of longer wavelengths, near the minimum loss wavelength of the MCF. As could be expected, the optimal position of the transmission band in the presence of XT is shifted towards shorter wavelengths due XT impact at longer wavelengths. However, we note that this shift is strongly dependent on the relative weights of the SNR contributions from XT, ASE noise and NLIN. As such, changes in the link characteristics would also modify the optimal operating wavelengths in the presence of XT. To illustrate this, Fig. 9(b) shows the optimal wavelength positions of the transmission bands with and without XT in the same conditions as Fig. 9(a) but using $100 \mathrm{~km}$ spans. In this case, the amount of ASE noise introduced in each span is higher, leading to a shift of the optimal operating wavelength range towards longer wavelengths, around $1555 \mathrm{~nm}$. In contrast, in the absence of XT, the optimal operating wavelengths remain unchanged.

Fig. 9(c) and (d) show the maximum throughput reached with and without XT as a function of the channel count. In both 

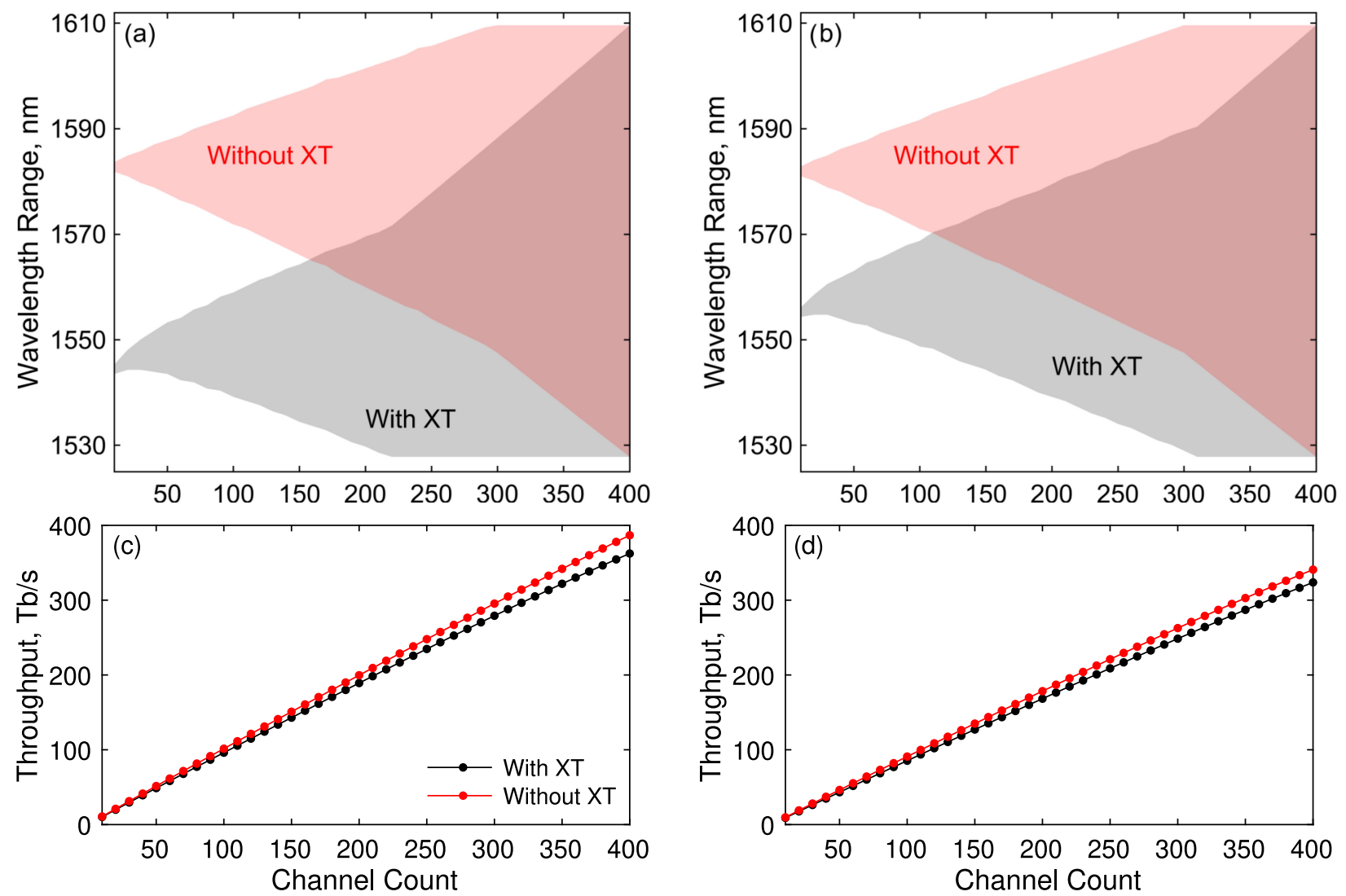

Fig. 9. The top sub-figures show the optimum wavelength range of the transmission band as a function of the channel count with and without XT on a 1600 km link with $80 \mathrm{~km}$ (a) and $100 \mathrm{~km}$ (b) spans, respectively. The bottom sub-figures show the maximum throughput as a function of the channel count assuming the optimized transmission wavelength range shown in the top sub-figures with and without crosstalk with $80 \mathrm{~km}(\mathrm{c})$ and $100 \mathrm{~km}(\mathrm{~d}) \mathrm{spans}$.

considered cases, the maximum throughput in the presence of XT was above $90 \%$ of the maximum throughput in the absence of XT. This result shows that this fiber could potentially be used for long distance transmission, as the overall XT-impact leads to less than $10 \%$ degradation of the maximum throughput. However, we should stress that for fibers with significantly higher XT, the increase of the achievable throughput with the channel count could be unable to compensate the corresponding increase in XT penalty. In such cases, it would be necessary to use XTmanagement techniques, such as the aforementioned direction interleaving [31].

\section{CONCLUSION}

This work presented an evaluation of the crosstalk-induced degradation of the signal-to-noise ratio in long-distance $\mathrm{C}$ and $\mathrm{L}$ band wavelength-division-multiplexing systems using homogeneous multicore fibers. It was shown analytically and experimentally that the crosstalk-induced penalty is independent on the transmission distance for sufficiently long uniform links. The wavelength dependence of crosstalk in multicore fibers was experimentally evaluated, showing nearly $10 \mathrm{~dB}$ tilt between the short edge of the C-band and the long edge of the L-band for the considered fibers. This tilt was experimentally shown to induce a crosstalk-induced degradation up to $3.8 \mathrm{~dB}$ for signals located near the long edge of the L-band. As a consequence, the design of wavelength-division-multiplexing systems using homogeneous multicore fibers must take into account the wavelength dependence of crosstalk. In this sense, this work presented a theoretical evaluation of the crosstalk-induced penalty as a function of the operating wavelength range and channel count in long-distance systems, taking also into account the wavelength dependence of fiber nonlinearities, including stimulated Raman scattering. It was shown that the crosstalk-impact forces the optimum operating wavelength towards the short C-band region, aroung $1545 \mathrm{~nm}$, in contrast with equivalent systems without crosstalk, which favor the low-loss wavelength region of the fiber, typically around $1580 \mathrm{~nm}$. Perhaps more significant was the observation that the optimum operating wavelength region for systems without crosstalk remained relatively unchanged when increasing the span length. In contrast the change in the contribution of amplified spontaneous emission noise resulting from an increase in span length lead to a reduction of the crosstalk-induced penalty, shifting the optimum wavelength operating range to higher values.

\section{ACKNOWLEDGMENT}

The authors would like thank the technical staff of the Photonic Network System Laboratory of the National Institute of Information and Communication Technology for the support with the experimental work. 


\section{REFERENCES}

[1] D. J. Richardson, J. M. Fini, and L. E. Nelson, "Space-division multiplexing in optical fibres," Nature Photon., vol. 7, no. 5, pp. 354-362, Apr. 2013.

[2] P. J. Winzer and D. T. Neilson, "From scaling disparities to integrated parallelism: A decathlon for a decade," J. Lightw. Technol., vol. 35, no. 5, pp. 1099-1115, Mar. 2017.

[3] H. Takara et al., "1.01-Pb/s (12 SDM/222 WDM/456 Gb/s) crosstalkmanaged transmission with $91.4-\mathrm{b} / \mathrm{s} / \mathrm{Hz}$ aggregate spectral efficiency," in Proc. 38th Eur. Conf. Exhib. Opt. Commun., Amsterdam, The Netherlands, Sep. 2012, Paper Th.3.C.1.

[4] B. J. Puttnam et al., " $2.15 \mathrm{~Pb} / \mathrm{s}$ transmission using a 22 core homogeneous single-mode multi-core fiber and wideband optical comb," in Proc. 41 st Eur. Conf. Exhib. Opt. Commun., Valencia, Spain, Sep. 2015, Paper PDP.3.1.

[5] B. J. Puttnam et al., " $0.715 \mathrm{~Pb} / \mathrm{s}$ Transmission over 2,009.6 km in 19-core cladding pumped EDFA amplified MCF link," in Proc. Opt. Fiber Conf., San Diego, CA, USA, Mar. 2019, Paper Th4B.1.

[6] M. Feuer et al., "Joint digital signal processing receivers for spatial superchannels," IEEE Photon. Technol. Lett., vol. 24, no. 21, pp. 1957-1960, Nov. 2012.

[7] B. J. Puttnam, R. S. Luis, J. M. Delgado Mendinueta, Y. Awaji, and N. Wada, "Linear block-coding across $>5 \mathrm{~Tb} / \mathrm{s}$ PDM-64qam spatial superchannels in a 19-core fiber," in Proc. EurConf. Opt. Commun., Valencia, Spain, Sep. 2015, Paper P.5.6.

[8] R. Luís et al., "Demonstration of a $1 \mathrm{~Pb} / \mathrm{s}$ spatial channel network node," in Proc.Eur. Conf. Opt. Commun., Dublin, Ireland, Sep. 2019, Paper PD3.5.

[9] T. Hayashi, T. Taru, O. Shimakawa, T. Sasaki, and E. Sasaoka, "Characterization of crosstalk in ultra-low-crosstalk multi-core fiber," J. Lightw. Technol., vol. 30, no. 4, pp. 583-589, Feb. 2012.

[10] J. Fini, B. Zhu, T. Taunay, M. F. Yan, and K. S. Abedin, "Statistical models of multicore fiber crosstalk including time delays," J. Lightw. Technol., vol. 30, no. 12, pp. 2003-2010, Jun. 2012.

[11] R. S. Luís et al., "Time and modulation frequency dependence of crosstalk in homogeneous multi-core fibers," J. Lightw. Technol., vol. 34, no. 2, pp. 441-447, Jan. 2016

[12] G. Rademacher et al., "Crosstalk dynamics in multi-core fibers," Opt. Express, vol. 25, no. 10, pp. 12 020-12 028, May 2017.

[13] R. S. Luís et al., "Spectral efficiency in crosstalk-impaired multi-core fiber links," in Proc. Shape Futur. Photon., San Francisco, CA, USA, Feb. 2018, vol. 1055102.

[14] R. S. Luís et al., "On the spectral efficiency limits of crosstalk-limited homogeneous single-mode multi-core fiber systems," in Proc. Adv. Photon., 2017, Paper NeTu2B.2.

[15] B. J. Puttnam et al., "Impact of intercore crosstalk on the transmission distance of QAM formats in multicore fibers," IEEE Photon. J., vol. 8, no. 2, Apr. 2016, Art. no. 0601109

[16] J. M. Gené and P. J. Winzer, "A universal specification for multicore fiber crosstalk," IEEE Photon. Technol. Lett., vol. 31, no. 9, pp. 673-676, May 2019.

[17] J. M. Gené et al., "Towards broadly optimum multi-core fiber designs," in Proc. Eur. Conf. Opt. Commun., Dublin, Ireland, Sep. 2019, Paper M.1.D.4.

[18] F. Ye et al., "Simple analytical expression for crosstalk estimation in homogeneous trench-assisted multi-core fibers," Opt. Express, vol. 22, no. 19 , Sep. 2014, Art. no. 23007.

[19] D. Semrau, R. I. Killey, and P. Bayvel, "A closed-form approximation of the gaussian noise model in the presence of inter-channel stimulated raman scattering," J. Lightw. Technol., vol. 37, no. 9, pp. 1924-1936, May 2019.

[20] T. Hayashi, T. Taru, O. Shimakawa, T. Sasaki, and E. Sasaoka, "Uncoupled multi-core fiber enhancing signal-to-noise ratio," Opt. Express, vol. 20 , no. 26, pp. B94-B103, Dec. 2012.

[21] D. J. Elson et al., "Analytical model for transmission performance of single mode multicore fibre with nonlinearity compensation," in Proc. 24th OptoElectron. Commun. Conf., Fukuoka, Japan, Jul. 2019, Paper MB1-3.

[22] P. Poggiolini, G. Bosco, A. Carena, V. Curri, Y. Jiang, and F. Forghieri, "The GN-model of fiber non-linear propagaion and its applications," $J$. Lightw. Technol., vol. 32, no. 4, pp. 694-721, Feb. 2014.

[23] D. Semrau, D. Lavery, L. Galdino, R. I. Killey, and P. Bayvel, "The impact of transceiver noise on digital nonlinearity compensation," J. Lightw. Technol., vol. 36, no. 3, pp. 695-702, Feb. 2018.

[24] R. S. Luís et al., "On the use of high-order mimo for long-distance homogeneous single-mode multicore fiber transmission," in Proc. Eur Conf. Opt. Commun., Gothenburg, Sweeden, Sep. 2017, Paper Th.2.F.2.
[25] R. S. Luís et al., "Long distance crosstalk-supported transmission using homogeneous multicore fibers and SDM-MIMO demultiplexing," Opt. Express, vol. 26, no. 18, pp. 24 044-24 053, Sep. 2018.

[26] A. Macho, M. Morant, and R. Llorente, "Experimental evaluation of nonlinear crosstalk in multi-core fiber," Opt. Express, vol. 23, no. 14, pp. 18 712-18 720, Jul. 2015.

[27] T. Hayashi, T. Taru, O. Shimakawa, T. Sasaki, and E. Sasaoka, "Design and fabrication of ultra-low crosstalk and low-loss multi-core fiber," Opt. Express, vol. 19, no. 17, Aug. 2011, Art. no. 16576.

[28] R. S. Luís et al., "Investigation of inter-core crosstalk and Raman nonlinearity in wideband MCF transmission," in Proc. Opt. Fiber Commun. Conf. Exhib., San Diego, CA, USA, Mar. 2017, Paper Th4H.6.

[29] G. Rademacher et al., "Experimental investigation of stimulated Raman scattering induced crosstalk-tilt in a homogeneous multi-core fiber," in Proc. Asia Commun. Photonics. Conf., Chengdu, China, Nov. 2019, Paper S3A.2.

[30] B. J. Puttnam et al., "Inter-core crosstalk penalties in wideband WDM, MCF transmission over transoceanic distances," in Proc. Eur. Conf. Opt. Commun., Rome, Italy, Sep. 2018, Paper Th1J.1.

[31] A. Sano, H. Takara, T. Kobayashi, and Y. Miyamoto, "Crosstalk-managed high capacity long haul multicore fiber transmission with propagationdirection interleaving," J. Lightw. Technol., vol. 32, no. 16, pp. 2771-2779, Aug. 2014.

[32] V. E. Perlin and H. G. Winful, "Optimal design of flat-gain wide-band fiber Raman amplifiers," J. Lightw. Technol., vol. 20, no. 2, pp. 250-254, Feb. 2002.

[33] T. Matsui et al., "118.5 Tbit/s transmission over $316 \mathrm{~km}-$ long multi-core fiber with standard cladding diameter," in Proc. Opto-Electron. Commun. Conf. Photon. Global Conf., Singapore, Jul. 2017, pp. 1-2.

[34] S. Papernyi et al., "To C- or not to C-, that is the question," in Proc. Eur. Conf. Opt. Commun., Dublin, Ireland, Sep. 2019, Paper Tu.1.A.6.

Ruben S. Luís (Member, IEEE) received the B.Sc. and M.Sc. degrees in electrical and computer engineering from Instituto Superior Tecnico, Technical University of Lisbon, Lisbon, Portugal, in 2000 and 2003, respectively, and the $\mathrm{Ph} . \mathrm{D}$. in electrical engineering from the University of Aveiro, Aveiro, Portugal, in 2007. Since 2016, he has been with the Photonic Network System Laboratory of the National Institute of Information and Communications Technology, Tokyo, Japan, where he researches advanced coherent transmission systems using spatial division multiplexing and optical packet switching.

Georg Rademacher (Senior Member, IEEE) was born in Minden, Germany, on July 23, 1986. He received the Dipl.-Ing. and Dr.-Ing. degrees from Technische Universität Berlin, Berlin, Germany, in 2011 and 2015, receptively. During his doctoral studies, he did internships with Bell Labs in Holmdel, New Jersey supported by the DAAD and NICT in Japan, supported by JSPS. Since 2016, he has a Researcher with NICT. He authored and coauthored more than 120 papers, published at international conferences or journals. His research focuses on systems and subsystems for high capacity transmission systems, primarily by applying space division multiplexing. He has received the 2017 ITG Award from the VDE, Germany and the Best Paper Award at the 2018 Photonics in Switching and Computing (PSC) Conference. He is a member of the IEEE Photonics Society and the VDE.

Benjamin J. Puttnam (Member, IEEE) received the M.Phys. degree in physics from the University of Manchester, Manchester, U.K., in 2000 and the Ph.D. degree from the University College London, London, U.K., in 2008. He is a Senior Researcher in the Photonic Network System Laboratory with the National Institute of Information and Communications Technology (NICT) in Tokyo, Japan. In between he worked as a Switch Design Engineer for T-mobile (U.K.). After short term visits to NICT, supported by JSPS and the Photonics Group with Chalmers University, Göteborg, Sweden supported by the Ericsson Research Foundation he re-joined NICT in March 2010. His current research interests are applications and sub-systems for SDM transmission and phase sensitive amplifier applications using PPLN waveguides. 
Daniel Semrau (Member, IEEE) received the B.Sc. degree in electrical engineering from the Technical University of Berlin, Berlin, Germany, in 2013, the M.Sc. degree in photonic networks engineering from Scuola Superiore Sant'Anna, Pisa, Italy, and Aston University, Birmingham, U.K., in 2015. He is currently working toward the Ph.D. degree with the Optical Networks Group, University College London, London, U.K. His research interests are mainly focused on channel modeling, nonlinear compensation techniques, and ultrawideband transmission coherent optical communications. He was the recipient of the Graduate Student Fellowship award of the IEEE Photonics Society, in 2018.

Robert I. Killey (Senior Member, IEEE) received the B.Eng. degree in electronic and communications engineering from the University of Bristol, Bristol, U.K., in 1992, the M.Sc. degree from University College London (UCL), London, U.K., in 1994, and the D.Phil. degree from the University of Oxford, Oxford, U.K., in 1998. He is currently a Professor of optical communications with UCL. He has participated in many European projects, including ePhoton/ONe, Nobel, BONE and ASTRON, and national projects. He is currently a Principal Investigator in the EPSRC funded UNLOC Project. His research interests include nonlinear fiber effects in WDM transmission, advanced modulation formats, and digital signal processing for optical communications. He was with the Technical Program Committees of many international conferences including European Conference on Optical Communication, Optical FiberCommunicationConference ACP, and OECC. He was an Associate Editor for the IEEE/OSA JOURNAL OF OPTICAL COMMUNICATIONS AND NETWORKING and is currently an Associate Editor for the Journal of Lightwave Technology.
Polina Bayvel (Fellow, IEEE) received the B.Sc. degree in engineering and Ph.D. degree in electronic and electrical engineering from University College London (UCL), London, U.K., in 1986 and 1990, respectively. In 1990, she was with the Fiber Optics Laboratory, General Physics Institute, Moscow, Russian Academy of Sciences, under the Royal Society Postdoctoral Exchange Fellowship. She was a Principal Systems Engineer with STC Submarine Systems, Ltd., London, U.K., and Nortel Networks (Harlow, U.K., and Ottawa, ON, Canada), where she was involved in the design and planning of optical fibre transmission networks. During 1994-2004, she held a Royal Society University Research Fellowship at UCL, where she became a Chair in Optical Communications and Networks. She is currently the Head of the Optical Networks Group, UCL, which she set up in 1994. She has authored or coauthored more than 300 refereed journals and conference papers. Her research interests include wavelength-routed optical networks, high-speed optical transmission, and the study and mitigation of fibre nonlinearities. She was the recipient of the Royal Society Wolfson Research Merit Award (2007-2012), IEEE Photonics Society Engineering Achievement Award in 2013, Royal Society Clifford Patterson Prize Lecture and Medal in 2014, and Royal Academy of Engineering Colin Campbell Mitchell Award in 2015. She leads the U.K. EPSRC Programme TRANSNET (2018-2024). She is currently a fellow of the Royal Academy of Engineering, Optical Society of America, and U.K. Institute of Physics. She is an Honorary Fellow of the Institution of Engineering and Technology.

Yoshinari Awaji biography not available at the time of publication.

Hideaki Furukawa (Member, IEEE) received the B.E., M.E., and Dr.Eng. degrees in material and life science from Osaka University, Osaka, Japan, in 2000 2002, and 2005, respectively. Since 2005, he has been with National Institute of Information and Communications Technology (NICT), Tokyo, Japan. From 2013 to 2014, he was the Deputy Director of the Ministry of Internal Affairs and Communications, Japan. His research interests include photonic information technology and photonic networks. He is a member of AKARI Architecture Design Project of NICT. 